\title{
CRITICAL THINKING: TOOLS FOR INTERNET INFORMATION EVALUATION
}

\author{
Mary Ann Fitzgerald \\ University of Georgia \\ Department of Instructional Technology \\ 607 Alderhold Hall \\ Athens, GA 30602 USA \\ E-mail: mfitzger@coe.uga.edu
}

\begin{abstract}
This qualitative study describes strategies employed by sophisticated adult World Wide Web users as they evaluate authentic Web information with the purpose of adapting these strategies for children in K-12 settings. The participants in this study followed think-aloud protocols and answered interview questions about two Web documents containing numerous misinformation devices. Evaluative strategies from these verbalizations were extracted and analyzed. Findings include a list of strategies and a description of three evaluative "styles." Finally, suggestions for the use and teaching of these strategies in elementary school through middle school are made.
\end{abstract}

\section{INTRODUCTION}

As World Wide Web access expands into schools and homes, children will likely encounter the misinformation often found in this medium. Are children, often alone and unsupervised, equipped to sort good information from bad? What specific vulnerabilities and dangers do they face? How can educators equip them with the evaluative skills they need to sift through this new wealth of information?, Purpose

The literature establishes misinformation as a potential problem for Internet users (Neavill, 1993; Viehland, 1993). The primary cause of this situation is an almost universal lack of gatekeeping and central authority. As a safeguard against nuclear attack, the Internet was built without a centralized controlling authority to distribute the information base throughout the United States (Stoker \& Cooke, 1995). Because of this lack of control "anybody can publish anything." (Neavill, 1984, p. 87) Without editorial control, documents flawed by bias, mistakes, lies, scholarly misconduct, or any of a number of other flaws, can circulate freely and instantly. Two related but less important causes of the misinformation problem are anonymity and hacking. Because the Internet allows anonymity, authors publishing misinformation fear no reprisals.

Hackers often break into government servers, intercepting, interrupting, and changing data without discovery (Stephens, 1995). If hackers can access data, they can change it. University servers, the most complete repositories of online information, are particularly vulnerable to hacker attack because of inadequate preventive measures (Coutorie, 1995).

Although censorship and technological screening have been suggested as possible solutions to the misinformation problem, the most practical approach is for readers to evaluate Web information themselves (Breivik, Senn, \& Gee, 1989). This issue is a practical as well as a philosophical one. It is unlikely that professional information managers can keep pace with new information on the Web in their efforts to index, evaluate, and screen information. Screening software, while effective to a degree, cannot filter out all objectionable material or misinformation. Even if these measures were totally effective, the philosophical and legal issues of freedom of speech and individual interpretations of truth present insurmountable dilemmas. For now, Internet users must recognize the need to do their own sifting and evaluating of Web information.

Are Internet users equipped with the skills they need for such a task? Critical skills to evaluate Web information may or may not be similar to those needed for general information literacy. Little is 
known about information evaluation in any medium, and existent studies indicate potential difficulties. Grice (1975) theorizes that people tend to believe that most information is true, and this theory harmonizes with Gilovich's assertion that people do not evaluate all incoming information for efficiency reasons (1991). At least two researchers have noted a remarkably passive acceptance of misinformation in memory studies (Belli, 1989; Highhouse \& Bottrill, 1995). Analyses Studies of scholarly misconduct demonstrate that have highlighted this problem in even the supposedly discerning academic community is prone to evaluative passivity arenas (Kochan \& Budd, 1992). Others writers have compiled case studies and long catalogs of successful hoaxes in the mass media hoaxes (Bird, 1996; Fedler, 1989; Tamarkin, 1993).

A review of psychological and social psychological literature reveals several disturbing possibilities about the effects of misinformation. People seem highly vulnerable to the manipulation of superficial presentation characteristics. An illustration of this fact is that subjects tend to accept without question information presented by a person perceived as having high status or expertise without question (Petty \& Cacioppo, 1986). In addition, readers rate texts with greater numbers of messages as being more believable than texts with fewer messages, regardless of message quality (Petty \& Cacioppoe, 1984). Audience members are more likely to accept arguments greeted with the enthusiasm of their fellow listeners (Axsom, Yates, \& Chaiken, 1987). In addition to these flaws in evaluative practice, people are vulnerable to the effects of misinformation may have disquieting manipulative effects upon memory. Loftus (1975) initiated a major trend in memory research with her discovery that witnesses of complex events exposed to conflicting misinformation after the event often reported the misinformation as part of the original memory. Anderson (1965) established that people tend to believe information presented first in a sequence, and disregard conflicting information presented later. These fragmentary and contradictory glimpses of how misjudgment and misinformation affects critical judgment and memory illustrate the fact that the poorly understood reader-misinformation interaction can potentially have profound results.

The few scholars who have studied misinformation in telecommunicated contexts report interesting findings. Aycock and Buchignani (1995), Hernon, (1995), and Viehland (1993)found a passive acceptance to misinformation in the electronic medium similar to that found by psychologists in other media. Sachs (1995) noted how the online discussion group he studied tended to reinforce the political biases of its readers. In addition, Aycock and Buchignani, as well as and Viehland, remarked upon how quickly and widely telecommunicated misinformation spread in their analyses of authentic online misinformation cases.

Several scholars and practitioners have published skill sets for online information evaluation (see, for example, Stripling \& Pitts, 1988; Weisburg \& Toor, 1994). Hernon (1995) asserts that Internet information is similar to information in other media in terms of quality. Thus, it is reasonable to assume that evaluative skill needed for online information differ little from skills needed for information in more traditional formats. However, this assumption has not been adequately tested. Also, as the literature shows, our knowledge of the thinking processes of readers as they encounter misinformation is fragmentary. One possible approach to this lack of knowledge is to examine the strategies of experts as they evaluate information. The purpose of this study is to describe the strategies used by sophisticated Web users as they make critical judgments about the quality of information found in authentic Web documents, and to adapt these strategies for use in K-12 settings.

For the purposes of this study, "misinformation" is defined as material presented as true although it contradicts facts presented in standard reference works. Information quality literature describes how authors can misrepresent facts through an array of linguistic tactics (see, for example, Lazere, 1982). These misrepresentations relate directly in some cases to the presentation manipulation findings described above. These linguistic tactics are labeled here as "devices of misinformation." The term "trigger" refers to the linguistic signal that marks the presence of a device. An information "problem" is a shortcoming of the information that can make it misleading, such as lack of currency or authority.

The following questions helped to shape the design of this study:

- What strategies identify effectively Web misinformation problems and devices? What clues (or "triggers") users to the presence of misinformation? What specific triggers are associated with specific devices? 
-What characteristics commonly identify "reputable" or "reliable" Web documents?

- What is the nature of Web misinformation? What devices do Web authors use to purposely misinform? How do these devices differ from those used in traditional communication, if at all?

- How can the strategies used by sophisticated adults be adapted for younger information users?

\section{METHOD}

Data collection consisted of Web document selection and downloading, interviews, and observations of expert participants as they explored authentic Web misinformation documents. The project began with the location, downloading, and careful examination of $23 \mathrm{Web}$ misinformation documents containing misinformation. Because it is difficult to escape the effects of personal bias in a study of this nature, A specific criterion was used during document selection. This selection criterion required that a document contain one or more of the devices of misinformation listed in previous work (Fitzgerald, 1997). (Still, it is necessary to admit that my personal beliefs ran strongly against the information contained in these documents.) Theoretically, information may contain devices of misinformation but still be valid. In general, however, authors of strong positions do not need to resort to such devices and avoid them because they lead to challenges from discerning readers. Therefore, misinformation devices are a fairly reliable means of identifying suspicious literature.

From these 23 documents, two were chosen for use in this particular project. The first document, which claimed that the Holocaust is a hoax and, contained a rich cataloging list of misinformation devices. The other document, a report attempting to legitimize parapsychology to support the commercial psychic service operating out of the same server, contained few devices but several major problems. Neither document was at all believable, in the researcher's opinion. Several other scholars and all participants in the study confirmed this assessment.

\section{PROCEDURE}

The hour-long audiotaped interviews and observations took place in a private office equipped with a networked Windows computer loaded with Netscape 3.0. Each session consisted of three phases: pre-interview, interactive evaluative task session, and post-interview. To begin, participants answered questions about pre-existing factors such as education, technological expertise, and bias about document topics. Next, they read each document in turn, followed think-aloud protocols as they read and answered assessment questions at completion. At the end of the session, the researcher debriefed each participant about the misinformation they had seen and answered any questions that occurred about the procedure.

The interactive task portion allowed participants to freely browse the two sample Web documents and simultaneously describe their thoughts. When the participant fell silent, or gave responses requiring further explanation, the researcher interrupted with probing questions. Participants were encouraged to talk as much as possible in a stream-of-consciousness manner. In addition, the researcher placed no limits upon the amount of time spent reading each document but explicitly stated that participants could read as little or as much of the document as they thought necessary. At completion, summary questions sought to uncovered specific judgments, reasoning processes, cognitive ambiguities, reasoning, and evaluative criteria.

\section{PARTICIPANTS}

Participants were recruited from personal colleagues at a major research university. Six graduate students, all with advanced Web expertise, participated in the study. Five of the students were enrolled in a doctoral educational technology program, while the sixth was completing a second master's degree in another field. All six had extensive experience with telecommunications and the Web. All but one were competent in constructing Web pages and writing HTML code. Three had participated in research projects directly related to Web information quality. Finally, four participants 
had backgrounds in the fields of communications, political science, advertising, and media production that gave them added expertise in media literacy. In sum, these participants were adequately qualified as Web information experts. Two were male and four were female. Ages ranged from 28 to 47.

It is important to consider pre-existing biases in relation to the subject matter of the two documents, the Holocaust and parapsychology. Without exception, participants believed in the reality of the Holocaust. Given the sensitive nature of the material to be examined, it was determined in advance that no participants were Jewish. Likewise, no participant believed strongly in parapsychology, although each one spontaneously indicated that they were open to its possibilities. It is quite possible that the summative evaluations of each participant are due as much or more to these pre-existing biases than to their evaluative strategies (Klayman \& Ha, 1987; Lord, Ross, \& Lepper, 1979). In the researcher's opinion, this limitation has little detrimental effect on the validity of the strategies voiced by each participant for two reasons. First, participants' verbalizations clearly demonstrate active strategy use. Second, it is also clear that four participants experienced mild disequilibrium when presented with provocative arguments and used, in part, evaluation strategies to relieve this disequilibrium.

\section{ANALYSIS}

The Web documents were analyzed thoroughly before interviewing began. This analysis prompted several probing questions asked after participants made their initial judgments. After three interviews, the researcher transcribed them and assessed methods and results. Because the interviews seemed to yield useful data, three more interviews were scheduled. Interview questions underwent minor revision during this assessment

After the completion and transcription of the interviews, all data, including interview transcripts, think-aloud transcripts, and observation notes, using open coding techniques, were analyzed. Next, the Web documents were reanalyzed after having reexamined the list of misinformation devices. During the second analysis, comparisons and parallels were drawn between devices found in the document, devices recognized by each participant, the triggers alerting participants to each device, and voiced evaluation strategies. Finally, member checks with four participants assured that strategies extracted from the transcripts matched participant recollections. In addition, a peer analyst, also an information expert, verified selected conclusions in a peer debrief. Trustworthiness of this data is further bolstered by the fact that most strategies appeared in the thinkalouds of more than one participant.

\section{FINDINGS}

This study yielded three major results. First, the misinformation presented fooled no participant in an overall way. Second, as hoped, participants revealed valuable information evaluation strategies as they voiced their thoughts about the material they were reading, valuable strategies were revealed. Finally, no participant noticed all of the devices used in the documents. In several cases, participants were misled to a minor degree by a particular device. This report focuses on the second finding. The third finding requires deeper analysis and more data collection, and will serve as the focus of a later report.

A summary of the misinformation devices and problems found, their definitions, associated triggers, and strategies used by participants to detect them is presented in Table 1. As they described their strategies, participants often contributed valuable insights gathered through experience. One new misinformation device appeared through this sharing of collective wisdom. This device, dubbed the "circular reference" by one participant, Rachel, is native to the Web and allows the author to quote herself or himself without seeming to do so. In both misinformation documents examined in this study, authors included numerous links to other documents on the same server. When followed, these links usually led to documents by the same author. Several participants pointed out this device, and declared that they seldom gave much credence to references to documents residing on the same server. Another valuable tip contributed by Paul is to visit the front end of the server by deleting the end of the URL in Netscape's "open" dialog box and pressing "return." Visiting the front end in 
this manner can help to establish useful facts about authority and purpose through identification of the sponsoring organization.

Participants shared several unique terms for triggering mechanisms. Henry called them "red flags." Rachel described the trigger process as "danger, danger, a little robin sings." In Table 1, where available, interesting participant phrases describe particular triggers. It is not always possible to associate a trigger with a specific device through the verbalizations of the participants, a frustrating artifact of this particular interview protocol. Finally, Table 1 lists strategies used by participants to uncover devices successfully. Several of these strategies are extremely broad-spectrumed, such as "use checklist."

Three evaluation "styles" were evident in addition to one overall trait. Two participants, Rachel and Paul, showed numerous signs of using a "checklist" approach. The primary marker of this approach was the naming of features sought before their appearance in the text. A superficial survey of the document before reading began also characterized this approach. One participant, Linda, seemed to use a more affective approach. Affective terms characterized her reaction, and she discussed the "tone" and the underlying emotions of the document. Interestingly, her approach has merit because she found more, different devices than any of the other participants. Another approach seemed to be the "global" approach. Henry described his approach with this term, saying that he weighed an entire document and tried to balance the good against the bad to arrive at an overall evaluation. A final trend that seemed evident over all six participants is that the presence of a single problem or device is extremely damaging to the credibility of the document.

\section{DISCUSSION}

\section{Limitations}

This project did not yield comprehensive results for three reasons. First, the documents chosen contain a limited set of devices. Thus, the list of strategies generated is not comprehensive for all devices of misinformation. Second, this study did not accomplished data saturation in six interviews, and thus more interviews should be conducted with additional participants and different documents. Finally, the participants in this study were aware that they would be encountering misinformation during the interactive Web task. The literature indicates that people significantly detect more misinformation when warned to search for it, but often accept it passively otherwise (Baker, 1979; Belli, 1989; Highhouse \& Bottrill, 1995). Markman (1979) and Markman and Gorin (1981) found a similar result with children. This forewarned condition was designed into the study, in order to isolate evaluative strategies from the problem of whether or not participants would recognize misinformation in the unprimed condition. Therefore, this study does not address a chief concern about misinformation.

Another obvious limitation is that findings based on data gathered from adults will be applied to children. However, at least one theorist asserts that the reasoning processes of children are essentially no different from that of adults, and children's reasoning errors are largely due to their lack of contextual knowledge (Carey, 1985). It is therefore reasonable to attempt a transfer of adult strategies to children. Evaluation of the outcomes of an instructional program based upon the results of this study may shed light upon Carey's theory.

\section{Interpretation}

Despite the limitations of this study, the results are useful. The data accomplished the primary purpose of strategy identification. Further, practical information contributed by the participants as well as TABLE 1 


\begin{tabular}{|c|c|c|c|c|c|c|}
\hline \multirow{4}{*}{ 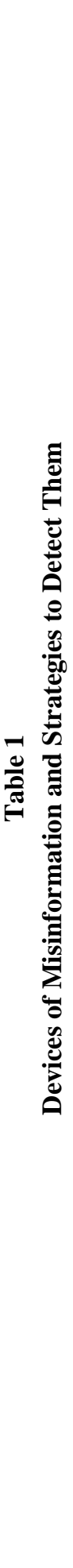 } & 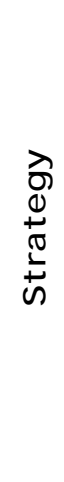 & 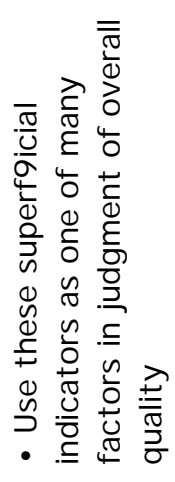 & 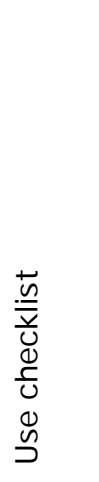 & 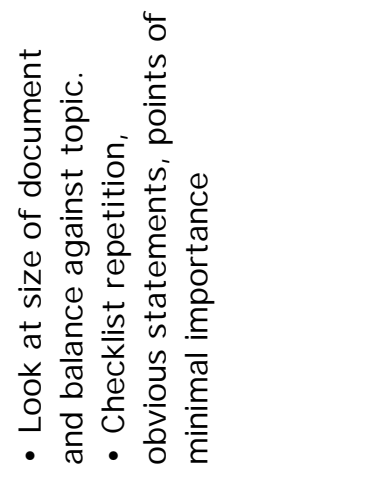 & 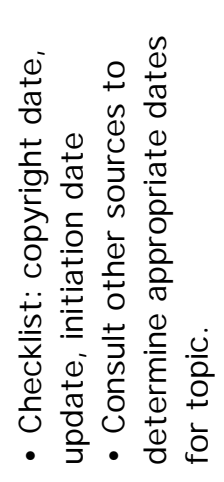 & 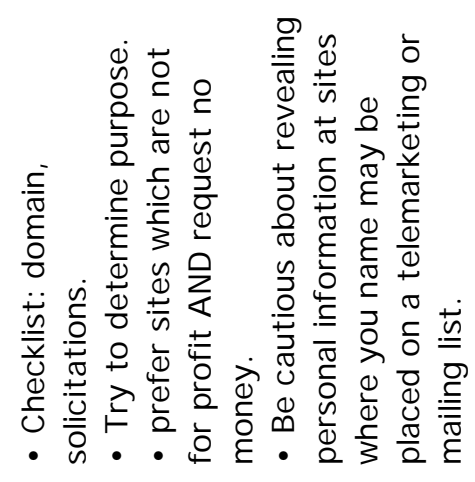 \\
\hline & 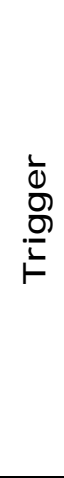 & 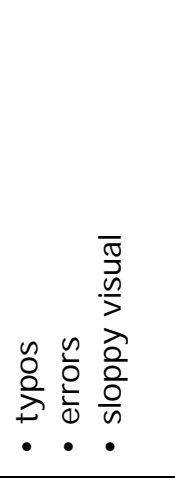 & 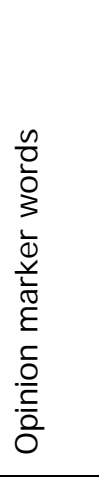 & 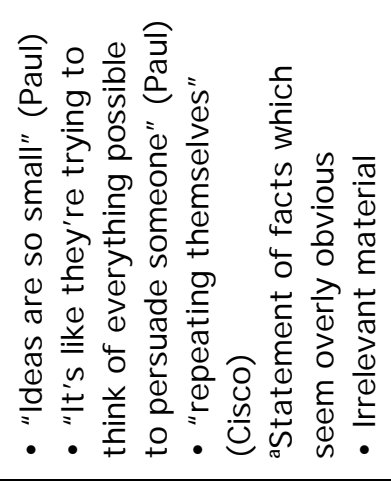 & 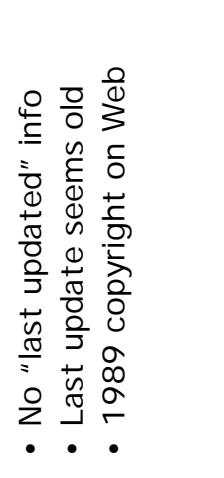 & 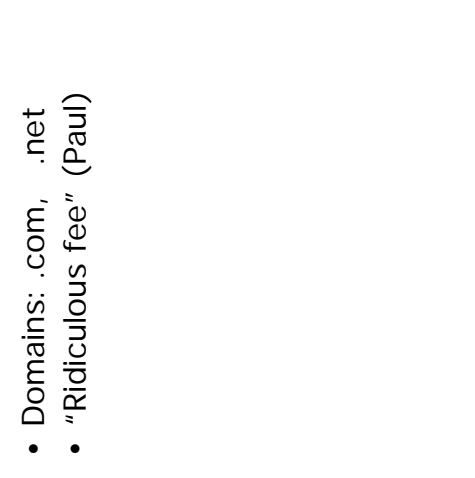 \\
\hline & 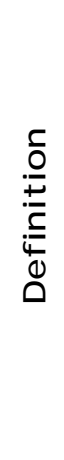 & 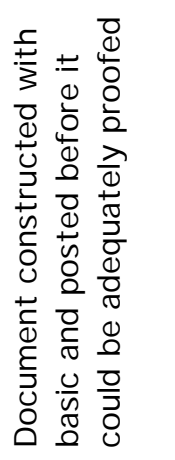 & 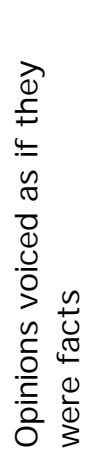 & 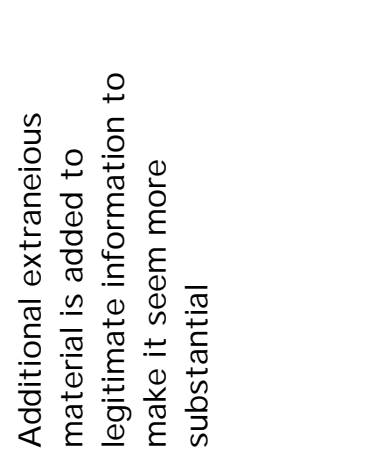 & 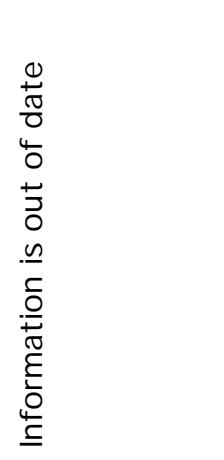 & 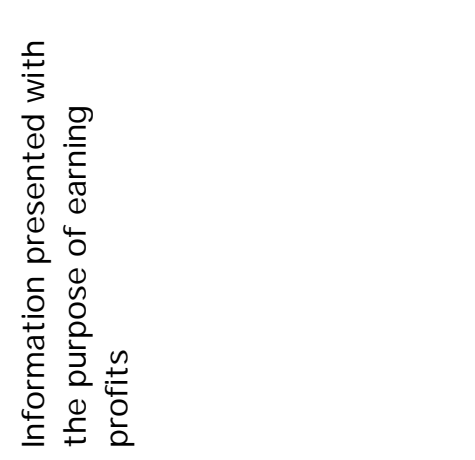 \\
\hline & 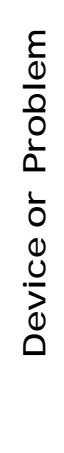 & 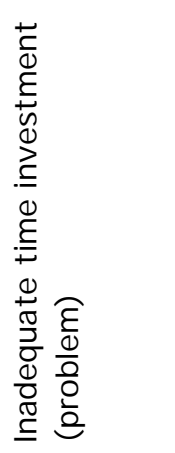 & 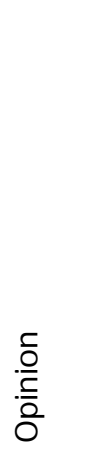 & 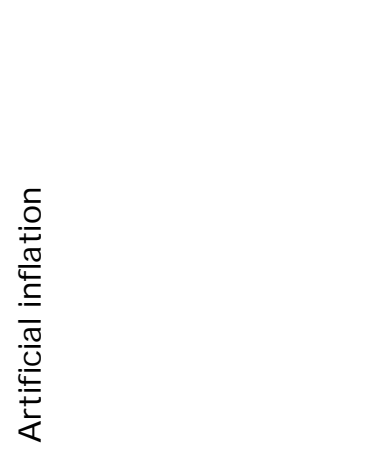 & 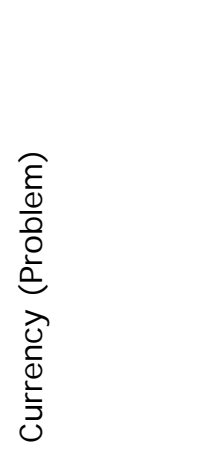 & 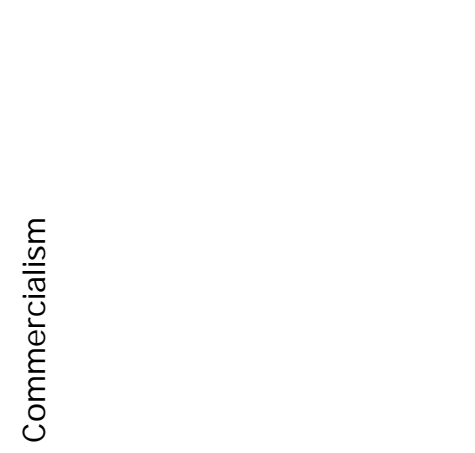 \\
\hline
\end{tabular}




\begin{tabular}{|c|c|c|c|c|c|}
\hline 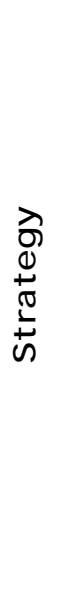 & 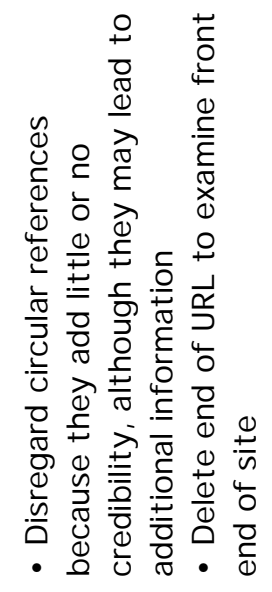 & 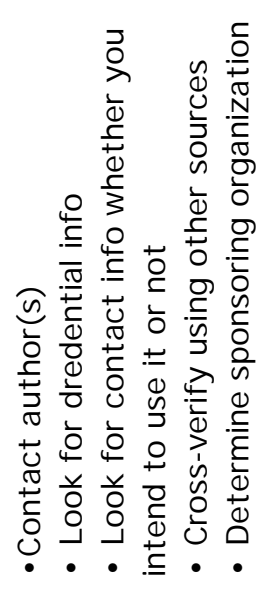 & 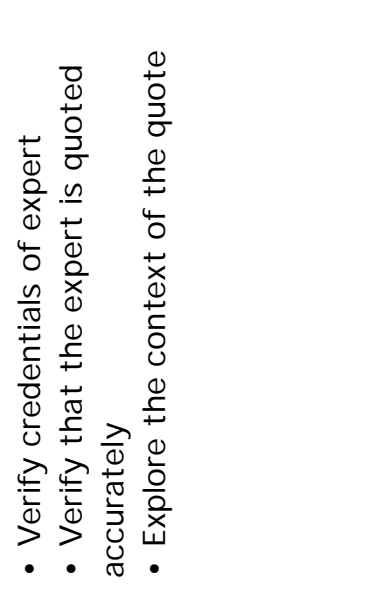 & 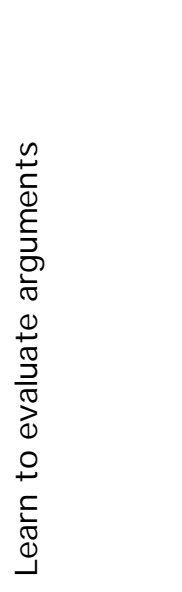 & 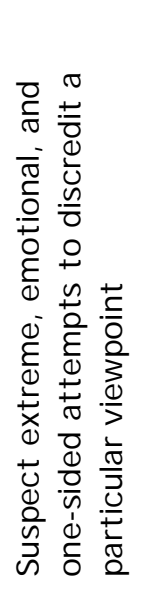 \\
\hline $\begin{array}{l}\frac{1}{0} \\
\text { סू } \\
\frac{0}{2} \\
\text { ㄴ }\end{array}$ & 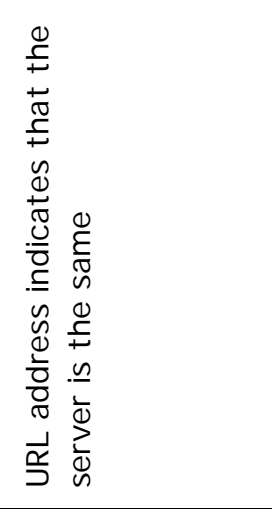 & 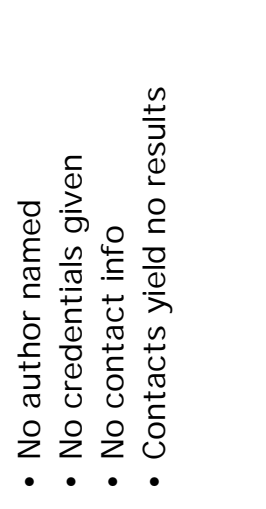 & 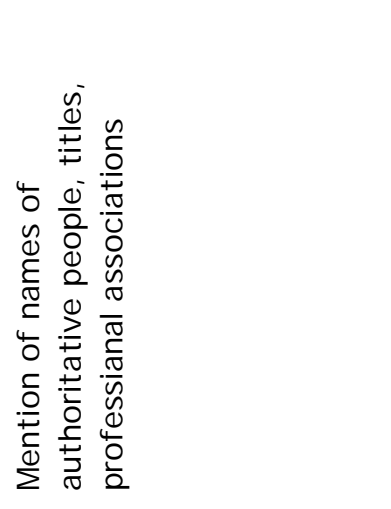 & 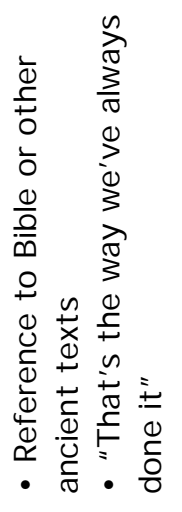 & 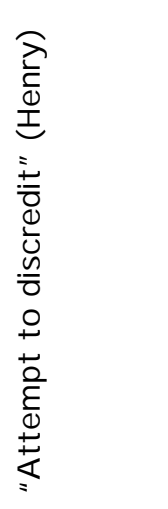 \\
\hline 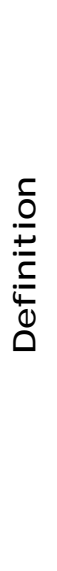 & 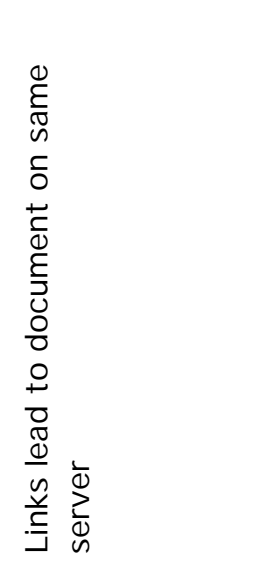 & 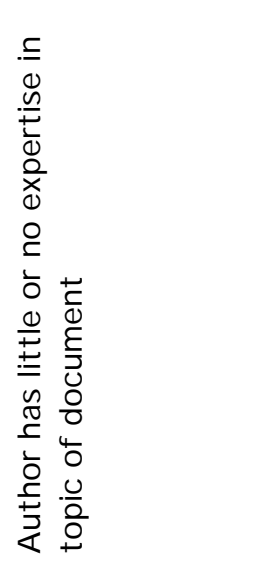 & 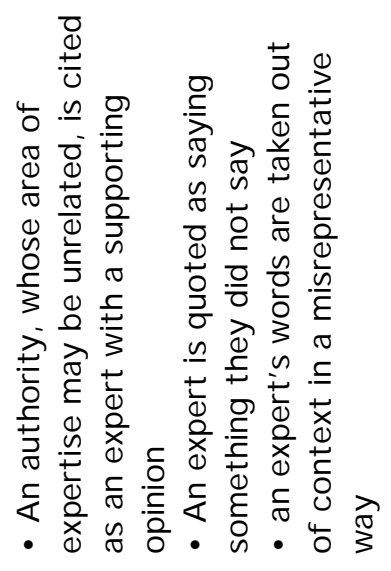 & 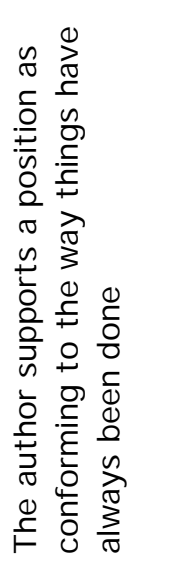 & 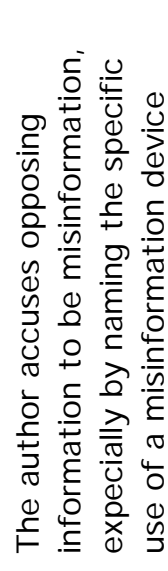 \\
\hline 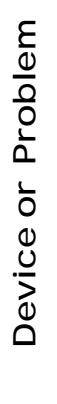 & 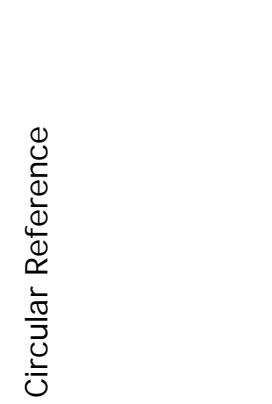 & 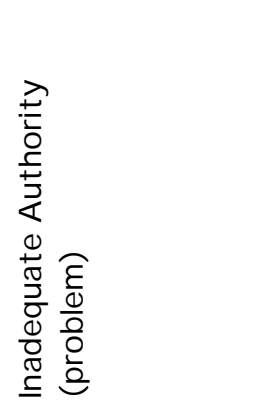 & $\begin{array}{l}\frac{t}{d} \\
\frac{0}{x} \\
\text { w }\end{array}$ & 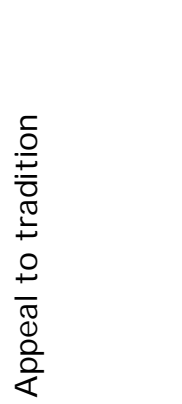 & 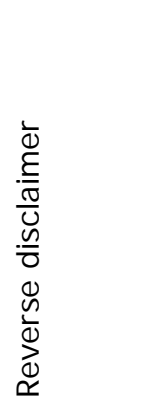 \\
\hline
\end{tabular}




\begin{tabular}{|c|c|c|c|c|c|}
\hline 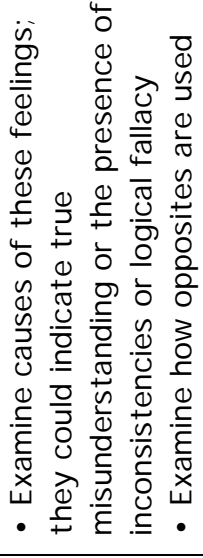 & 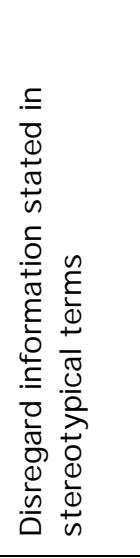 & 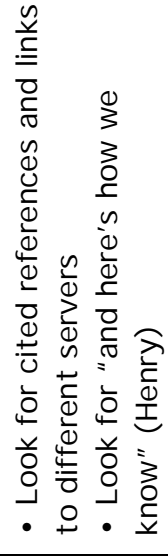 & 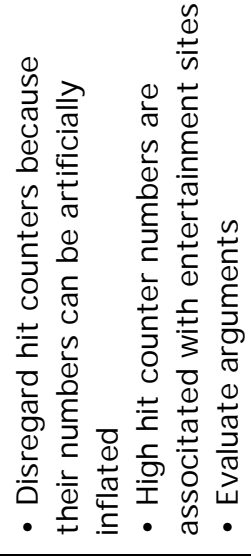 & 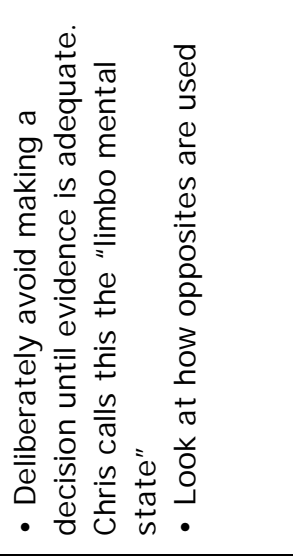 & 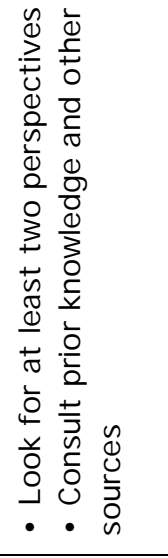 \\
\hline 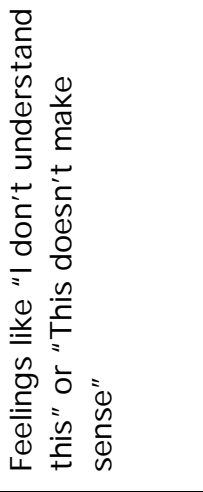 & 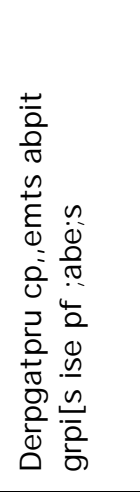 & 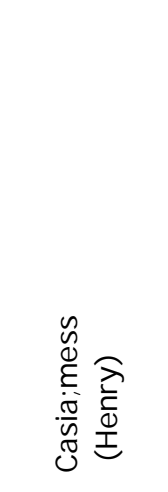 & 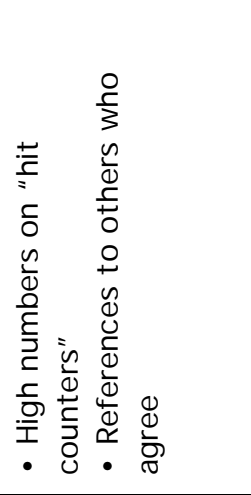 & 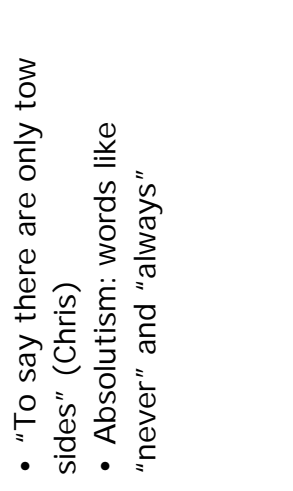 & 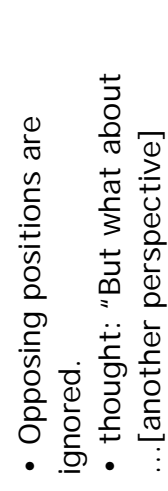 \\
\hline 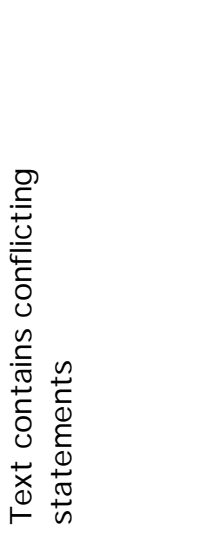 & 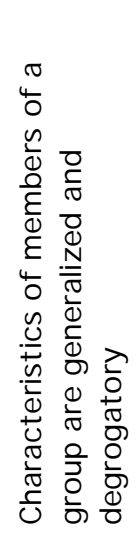 & 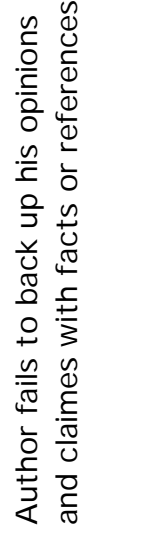 & 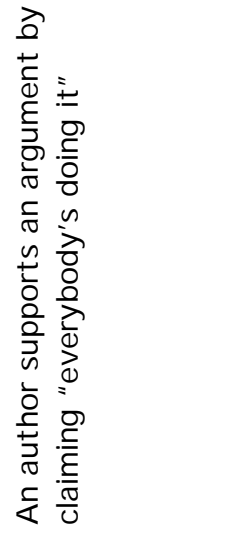 & 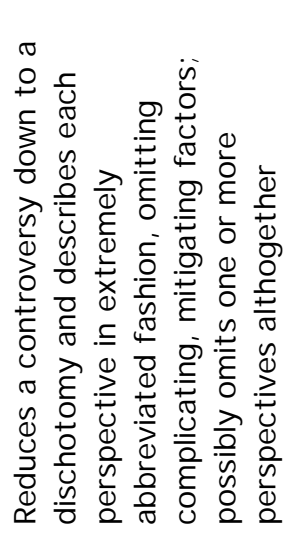 & 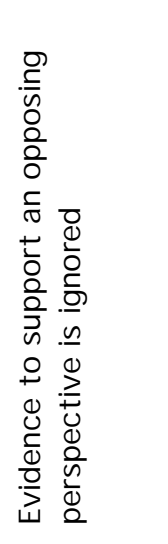 \\
\hline 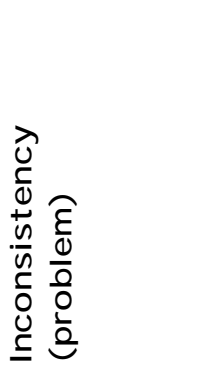 & 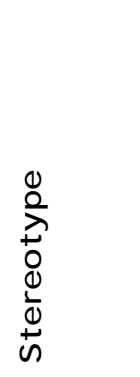 & 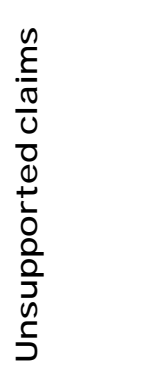 & $\begin{array}{l}5 \\
0 \\
0 \\
0 \\
3 \\
0 \\
\frac{5}{10} \\
0\end{array}$ & 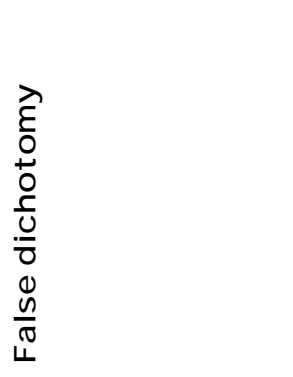 & 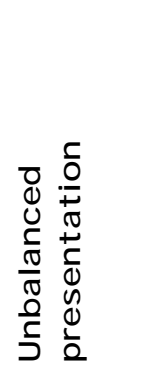 \\
\hline
\end{tabular}


information about their evaluative styles may enhance the application of evaluative strategies in K-12 settings.

The checklist approach is an excellent and easily implemented starting point for information evaluation. Several good checklists are available (Schrock, 1996a, 1996b, 1996c; Wilkinson, Bennett, \& Oliver, in press et al; Quinlan). However, many of the strategies used by participants in this study do not fit easily into the "yes, it's here/no, it's not here checklist approach. Therefore, the checklist heads a list as one of several strategies, all of which should be used in evaluation (Table 2). The emotional approach merits attention also, because sensitivity to the emotions expressed in a document may reveal devices and problems like stereotyping, bias, and emotional manipulation. The other evaluative style, global evaluation, and the tendency of the participants to discount an entire document on the basis of a single problem do not seem appropriate for K-12 application.

Hernon (1995) asserts that information on the Internet is "no better or worse" than information in any other medium (p. 136). This notion is intuitively countered by the probability that misinformation will continue to flourish due to the lack of gatekeepers on the Web. In corroboration of this theory, participants in this study all remarked about the high percentage of lowquality information on the Web. Rachel expressed the gatekeeper issue in these words: "The main difference is, with a book you know there had to be an editor at some point...[on the Web] you have to be your own editor." Chris expressed concerns about how easily electronic information can be altered, and raised the issue of political revision similar to that in Orwell's classic novel 1984 (1949). Linda talked at length about differences in cognitive reception of information between the Web and other media:

If you've got something that's printed..you've got to do something. You have to throw it in the trash, you have to put it back, it's in your hand. So it's a little bit more tangible...When you're on the Web, you just [click] and it's gone. So much easier, so much faster, so much more immediate...In a way maybe that's a good thing because you're out of there really fast; but maybe that's a bad thing because as you're carrying this back to throw it in the trash or put it away you're reaffirming the fact that this is not the right information.

This former primary school teacher's observation resonates with the concrete operations theories of Piaget (1948).

The participants in this study constructed their strategies over years of practice and study. These strategies, carefully selected and adapted according to pedagogical principles, can be taught to children. Drawing upon the researcher's own K-12 teaching experience and the writings of educational theorists, suggested teaching strategies are listed below.

\section{APPLICATION}

Teaching evaluative strategies to children is challenging. Children cannot be instructed to search for abstract constructions like bias and logical fallacies without extensive preparation. Children of elementary school age are also uncomfortable with the disequilibrium caused by ambiguity, a necessary accompaniment to evaluative thinking (Piaget, 1948).

Another issue to be addressed is a philosophical one. At what age is it appropriate to teach children that people sometimes lie? Many parents and teachers believe that children should learn to trust adults and obey authority, and that discussions about lying will undermine this teaching. On the other hand, children witness untruths, fiction, and fantasy every day in many different media. More ominously, people eager to exploit the naive and the young through commercial and criminal means stalk the Internet. This troubling issue is far beyond the scope of this report, but teachers and parents must consider it before applying interventions for misinformation detection.

Specific strategies suitable for K-12 students appear in Table 2. Because of the complexity of several of these strategies, recommended grade levels for introduction are included. In addition, many of these strategies occur in varying levels according to the demands of the text. The teacher is the best judge of TABLE 2 


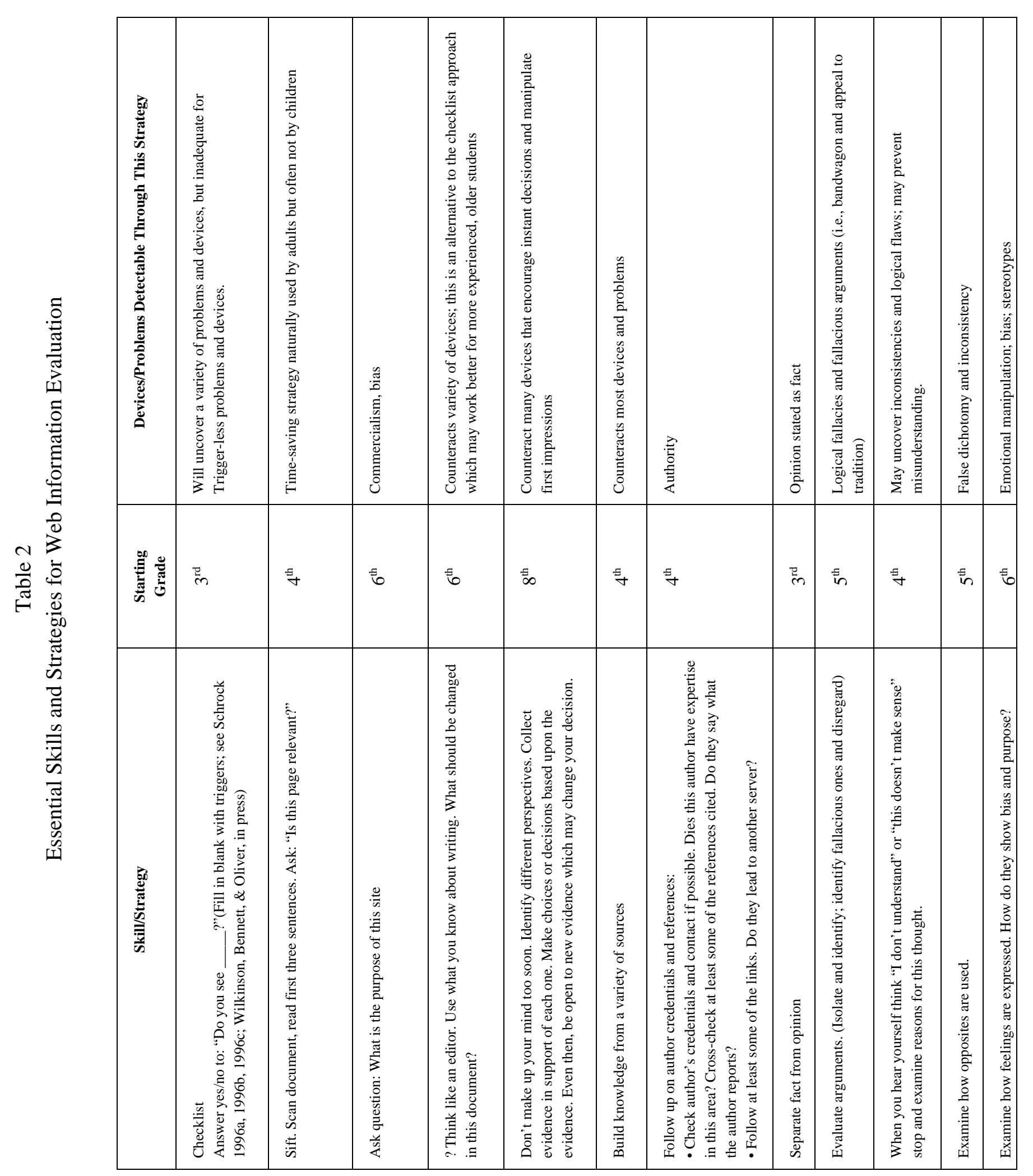


when and how to apply differing levels of difficulty. Most of the strategies, especially argumentation, should be taught over a span of years.

It is vitally important that information literacy skills in general and these strategies in particular be taught in the context of subject matter material (Callison, 1993). Luckily, critical thinking strategies fit well with most content areas. Few topics could be more boring or incomprehensible to children than critical thinking or argumentation taught out of context. The best approach is to choose a subject area of current, controversial interest to the students in a given class, and integrate the suggested strategies into a unit about that topic. For example, a current educational controversy is whether or not school children should wear uniforms. People with fervent opinions speak hotly on both sides of this issue, and students feel powerlessly caught between. Opinions on this topic appear in newspapers, broadcast media, the Web, and Internet discussion forums. Students could explore this issue through all of these media. In the course of doing so, misinformed opinions and false information are bound to surface, and the strategies listed in Table 2 will necessarily come into play. This research should culminate in some public forum such as a debate or a special issue of the school newspaper. Projects such as these require a great deal of planning and time on the part of teachers and media professionals, but Dewey (1915) recommends them as an extremely most effective type of learning.

\section{CONCLUSION}

The series of qualitative interviews reported in this paper explored questions relating to Web misinformation and strategies for detecting such misinformation. Expert Web users served as a source of strategies for others who can benefit from their experience. Suggestions for adapting, applying, and teaching these strategies in K-12 contexts were made. Although these strategies may seem straightforward at first glance, the pedagogical and philosophical issues involved in teaching them to children include complicated pedagogical and philosophical issues. Nevertheless, educators must address these issues to prepare the students of today for their futures as information consumers.

This area of inquiry provides many opportunities for future research. Interviews of more experts with different documents containing different devices should reveal new strategies keyed toward specific devices. In addition, a repertoire of several strategies may better serve the different learning styles represented among different individuals. Two other vital but inadequately explored issues involve the spontaneous application of critical thinking and the long-term effects of misinformation upon memory. Presently, we know very little about why people decide to use evaluative skills in a given situation, provided that they have such skills. While psychologists have studied misinformation effects in respect to memory, few studies have addressed misinformation effects in an educational sense. The World Wide Web presents an excellent medium through which to study all of these issues.

While we know few facts about the effects of Web misinformation upon people, it seems likely that more misinformation will be published on the Web than in any other medium except for spoken communication unless some agency intervenes and begins to "police" the Web. As the only truly democratic medium except for spoken communication, such policing would spoil a valuable public resource. As it stands, educators should view the Web as an opportunity to further the vital critical thinking agenda. In the meantime, it is imperative that scholars and educators learn more about the effects of misinformation.

Debate continues over how the educational establishment can best foster the intertwined skills of critical thinking and information literacy. This research should contribute to educational efforts by describing strategies that successfully detect misinformation. Further, this exposure and analysis of Web misinformation samples may awaken users to the importance of critical evaluation and help to encourage the spontaneous application of critical reasoning.

\section{REFERENCES}

Anderson, N. H. (1965). Primacy effects in personality impression formation using a generalized order effect paradigm. Journal of Personality and Social Psychology, 2 (1), 1-9.

Axsom, D., Yates, S. M., \& Chaiken, S. (1987). Audience response as a heuristic clue in persuasion. Journal of Personality and Social Psychology, 53 (1), 30-40. 
Aycock, A., \& Buchignani, N. (1995). The e-mail murders: Reflections on 'dead' letters. In S.G. Jones (Ed.), Cybersociety (pp. 184-231). Thousand Oaks, CA: SAGE.

Baker, L. (1979). Comprehension monitoring: Identifying and coping with text confusions. Journal of Reading Behavior, 11 (4), 365-374.

Belli, R. F. (1989). Influences of misleading postevent information: Misinformation interference and acceptance. Journal of Experimental Psychology: General, 118 (1), 72-85.

Bird, S. E. (1996). CJ's revenge: Media, folklore, and the cultural construction of AIDS. Critical Studies in Mass Communication, 13 (1), 44-58.

Breivik, P. S., Senn, P., \& Gee, E. G. (1989). Information literacy. New York: Macmillan.

Callison, D. (1993). Expanding the evaluation role in the critical-thinking curriculum. In C. C. Kuhlthau (Ed.), School library media annual (pp. 43-57). Englewood, CO: Libraries Unlimited.

Carey, S. (1985). Are children fundamentally different kinds of thinkers and learners than adults? In S.F. Chipman \& J.W. Segal (Eds.), Thinking and learning skills, Vol. 2 (pp. 485-517). Hillsdale, NJ: Lawrence Erlbaum.

Coutorie, L. E. (1995). The future of high-technology crime: A parallel Delphi study. Journal of Criminal Justice, 23 (1), 13-27.

Dewey, J. (1915). The school and society. Chicago: University of Chicago Press.

Fedler, F. (1989). Media hoaxes. Ames, IA: Iowa State University Press.

Fitzgerald, M. A. (1997). Misinformation on the Internet: Applying evaluation skills to online information. Emergency Librarian, 24 (3), 9-14.***

Gilovich, T. (1991). How we know what isn't so: The fallibility of human reason in everyday life. New York: Free Press.

Grice, H.P. (1975). Logic and conversation. In D.Davidson \& G. Harman (Eds.), The logic of grammar (pp. 64-75). Encino, CA: Dickenson.

Hernon, P. (1995). Disinformation and misinformation through the Internet: Findings of an exploratory study. Government Information Quarterly, 12 (2), 133-139.

Highhouse, S, \& Bottrill, K.V. (1995). The influence of social (mis)information on memory for behavior in an employment interview. Organizational Behavior and Human Decision Processes, 62 (2), 220-229.

Klayman, J., \& Ha, Y.W. (1987). Confirmation, disconfirmation, and information in hypothesis testing. Psychological Review, 94 (2), 211-228.

Kochan, C.A., \& Budd, J.M. (1992). The persistence of fraud in the literature: The Darsee case. Journal of the American Society for Information Science, 43 (7), 488-493.

Lazere, D. (1982). Composition for critical thinking: a course description. (ERIC Document Reproduction Service No. ED 273 959).

Loftus, E.F. (1975). Leading questions and the eyewitness report. Cognitive Psychology, 7 (4), 560572 .

Lord, C.G., Ross, L., \& Lepper, M.R. (1979). Biased assimilation and attitude polarization: The effects of prior theories on subsequently considered evidence. Journal of Personality and Social Psychology, 37 (11), 2098-2109.

Markman, E.M. (1979). Realizing that you don't understand: Elementary school children's awareness of inconsistencies. Child Development, 50 (3), 643-655.

Markman, E.M., \& Gorin, L. (1981). Children's ability to adjust their standards for evaluating comprehension. Journal of Educational Psychology, 73 (3), 320-325.

Neavill, G.B. (1984). Electronic publishing, libraries, and the survival of information. Library Resources and Technical Services, 28 (1), 76-89.

Neavill, G.B. (1993). Libraries and texts in the electronic environment. In R.S. Martin (Ed.), Scholarly communication in an electronic environment (pp. 53-69). Chicago: American Library Association.

Orwell, G. (1949) 1984. New York: Harcourt, Brace.

Petty, R.E., \& Cacioppo, J.T. (1984). The effects of involvement on responses to argument quantity and quality: Central and peripheral routes to persuasion. Journal of Personality and Social Psychology, 46 (1), 69-81. 
Petty, R.E., \& Cacioppo, J.T. (1986). The elaboration likelihood model of persuasion. In L. Berkowitz (Ed.), Advances in experimental social psychology, (Vol. 19, pp. 123-205). Orlando, FL: Academic Press.

Piaget, J. (1948). Judgment and reasoning in the child. New York: Harcourt, Brace.

Sachs, H. (1995). Computer networks and the formation of public opinion: An ethnographic study. Media Culture and Society, 17 (1), 81-99.

Schrock, K. (1996a). Critical evaluation survey: elementary school level. [On-line]. Available: <http://www.capecod.net/Wixon/evalelem.htm>.

Schrock, K. (1996b). Critical evaluation survey: middle school level. [On-line]. Available: <http://www.capecod.net/Wixon/evalmidd.htm>.

Schrock, K. (1996c). Critical evaluation survey: secondary school level. [On-line]. Available: <http://www.capecod.net/Wixon/evalhigh.htm>.

Stephens, G. (1995). Crime in cyberspace. Futurist, 29 (5), 24-28.

Stoker, D., \& Cooke, A. (1995). Evaluation of networked information sources. In A.H. Helal \& J.W. Weiss (Eds.), 17th International Essen Symposium: Information superhighway: the role of librarians, information scientists, and intermediaries (pp. 287-312). Essen, Germany: Essen University Press.

Stripling, B. K., \& Pitts, J. M. (1988). Brainstorms and blueprints: Teaching library research as a thinking process. Englewood, CO: Libraries Unlimited.

Tamarkin, B. (1993). Rumor has it: A curio of lies, hoaxes, and hearsay. New York: Prentice Hall.

Viehland, D.W. (1993). Dear Mr. President: A story of misinformation distribution in cyberspace. Internet Research, 3 (3), 57-60.

Weisburg, H.K., \& Toor, R. (1994). Learning, linking and critical thinking. Berkeley Heights, NJ: Library Learning Resources.

Wilkinson, G.L., Bennett, L.T., \& Oliver, K.M. (in press). Evaluation criteria and indicators of quality for Internet resources. Educational Technology. 